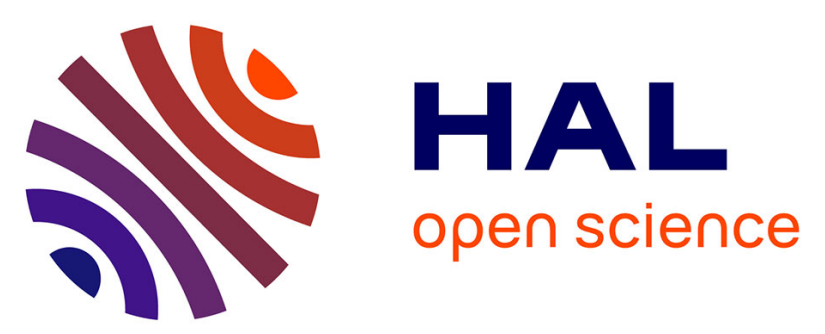

\title{
Panorama of real-life applications in logistics embedding bin packing optimization algorithms, robotics and cloud computing technologies
}

\author{
Abderrahmane Aggoun, Ahmed Rhiat, François Fages
}

\section{- To cite this version:}

Abderrahmane Aggoun, Ahmed Rhiat, François Fages. Panorama of real-life applications in logistics embedding bin packing optimization algorithms, robotics and cloud computing technologies. GOL'16 - 3rd IEEE International Conference on Logistics Operations Management, May 2016, Morocco, Morocco. 10.1109/GOL.2016.7731693 . hal-01378469

\author{
HAL Id: hal-01378469 \\ https://hal.science/hal-01378469
}

Submitted on 30 Nov 2016

HAL is a multi-disciplinary open access archive for the deposit and dissemination of scientific research documents, whether they are published or not. The documents may come from teaching and research institutions in France or abroad, or from public or private research centers.
L'archive ouverte pluridisciplinaire HAL, est destinée au dépôt et à la diffusion de documents scientifiques de niveau recherche, publiés ou non, émanant des établissements d'enseignement et de recherche français ou étrangers, des laboratoires publics ou privés. 


\section{Panorama of real-life applications in logistics embedding bin packing optimization algorithms, robotics and cloud computing technologies}

\author{
Abderrahmane AGGOUN \\ KLS OPTIM \\ 53, rue des Casseaux \\ 91140 Villebon sur Yvette, France \\ +33160146685 \\ Abder.Aggoun@klsoptim.com
}

\author{
Ahmed RHIAT \\ ICAM de Lille \\ 6 , rue Auber \\ 59046 Lille, France \\ +33 320226385 \\ Ahmed.Rhiat@icam.fr
}

\author{
François FAGES \\ INRIA Paris-Rocquencourt \\ Domaine de Voluceau Team Lifeware \\ 78150 Rocquencourt, France \\ +33139635709 \\ Francois.Fages@inria.fr
}

\begin{abstract}
In this paper, we are interested in one hand to review a set of problems encountered in logistics, and in a second hand to highlight the contribution of Constraint Programming, Metaheuristics and numerical solvers using non-linear inequalities in solving them. One of the objectives is to address a panorama of bin packing applications in logistics and their embedding in cloud computing.
\end{abstract}

\section{Keywords}

Optimization; Constraint Programming; Bin Packing; Metaheuristics; Cloud Computing; Operational Logistics; IOT; Robotics; RFID.

\section{INTRODUCTION}

Problems addressed are taken from production, warehousing and distribution. In production, typical problems are the design of optimal packing plans of identical items in containers. In the article we present successful real-word applications using state of the art of advanced optimization algorithms where optimality is often required. The article also presents current state of the art of dealing with complex shapes where the problems to solve are still hard to solve and the exploitation of optimization algorithms is not trivial. In warehousing and distribution, problems addressed concern order preparation of large orders for intermediate platforms or important customers like manufacturers in different sectors. An order is a set of lines. Each line describes the ordered product with its corresponding quantity. The problem consists of packing products of various shapes and sizes into available pallets (large boxes) in a way which optimizes the total number of pallets. The objective is to minimize the total number of pallets whist handling several types of pallets and subject to orientation, fragility, stackability, weight and volume constraints. All these problems are known as bin packing problems $[3,5]$. Bin packing algorithms were extended and applied to operational planning of trains. To come up with working solutions, we developed several optimization solvers combining several technologies: Constraint Programming, Metaheuristics like tabu search or specific solvers for handling complex shapes with non-linear inequalities. Solvers are integrated in Full Web applications and Cloud Computing platforms, many of them have been operational for many years. In this article we also present preliminary results of integration of robotics, drones and optimization and the potential applications of such results in production, warehousing and e-commerce.

\section{Constraint Programming}

Constraint Programming (CP) [2] has become a reality in the industrial world. Its success represents an important development of Artificial Intelligence and a successful vulgarization of the techniques of Operations Research in decision-making solutions in various domains. Indeed, CP has effectively addressed a large class of combinatorial problems. The Constraint Programming system used is Choco [http://choco.emn.fr] designed to tackle real world "constrained search" problems with a short-term development time and good efficiency. The Choco system provides a number of constraint solvers over different computation domains. These domains have been chosen because of their interest for applications, but also since efficient specialized constraint solving methods exist for these domains. Constraint Programming is based on the idea that many interesting and difficult problems can be expressed declaratively in terms of variables and constraints. The variables range over a (finite) set of values and typically denote alternative decisions to be taken. The constraints are expressed as relations over subsets of variables and restrict feasible value combinations for these variables. A solution is an assignment of values (search) to variables, which satisfies all constraints. It is the ability of $\mathrm{CP}$ systems to take into account heterogeneous constraints effectively which, in the $90 \mathrm{~s}$, raised a commercial interest in this paradigm.

\section{Bin packing optimization solutions}

\subsection{Optimization of pallets}

The problem addressed is taken from warehousing and distribution. It concerns the order preparation of large orders for intermediate platforms or important customers like manufacturers in different sectors. An order is a set of lines. Each line describes the ordered product with its corresponding quantity. A carton in a pallet may have up to six possible orientations which may increase the complexity in some cases. The problem consists of packing boxes subject to orientation, fragility, stackability, weight and volume constraints of various sizes into available pallets (larges boxes) in a way which optimizes the total number of pallets. The objective is to minimize the total number of pallets.

The type of planning is operational planning which means that some decisions must be taken in real time. The context is the following. The in-house warehouse management system (WMS) is connected to an ERP (SAP). The WMS reads the orders to prepare each lap of time (e.g. 1 minute) from the ERP. The main constraint is that the WMS must compute the optimal number of pallets in real time and the optimal linear meters of the virtual vehicle. This decision making information is sent in real time to the transporters (if they are known) or to the transport department. The objective is to compute this decision making information in just a few seconds. For example, the decision is taken at 09:00 and the preparation will start at 15:00. Usually the exact number of pallets is known once the order preparation is finished. In this case, the consequence is that the transport is informed at the last minute with many disadvantages; e.g. sending a large vehicle by using half of the vehicle capacity. We have noticed that objectives and requirements 
are subject to many changes depending on the final client. In the same time the number of different sizes of boxes increases each year. Therefore we developed many parametric variants of solvers with different strategies like packing by layers, by piles or a combination of both. A CP program is structured in three parts: declaration of decision variables, statement of constraints and enumeration. Constraint Programming allows to separate the modeling of heuristics and strategies from constraints. The search combines constructive solution procedures and some CP strategies. It takes advantage of the constraint programming propagation (domain of variables is reduced after each step of assignment) and the strategies used to select each step, the best object (container, configuration), the best decision variable and the best value. Search procedures make it possible to find a compromise between optimal solution and quality solutions which are preferred in practice. Different variants of optimizations of pallets are deployed in various sectors: automotive, perfume industries, pharmacy, foodprocessing, chocolate factory, etc.

\subsection{Vehicle / Container Loading Plans}

Optimization of vehicle loading is a well-known problem in TMS (Transport Management Systems). For some sector activities in logistics they are complementary to pallet optimization. For some variants the combinatorial problems to solve are similar. Furthermore, one can find similar constraints. First we distinguish two types of problems: (1) a single container loading problem (SCLP): Boxes of different sizes are stowed in the container as is possible by minimizing the unused. The objective is to maximize the use of a container's volume; (2) a multiple container loading problem (MCLP): Boxes of different sizes are stowed in several containers. The objective is to minimize the total number of required containers. Optim Vehicle / Truck tool provides several engine solvers. Each engine solver is dedicated to a variant of SCLP or MCLP problems. There are different variants of solvers for vehicle loading problems. For example, in some cases the loading is carried out in two steps. First one has to build pallets or piles (grouping compatible items) and then to optimize their loading into vehicles. The number of items to pack may vary from about ten to several thousand. Like the optimization of pallets, the search procedure is a key to compute quality solutions under time constraint.

\subsection{Bin packing e-commerce \\ 3.4 Applications}

This application is a variant of the optimization of pallets where items are products (small cartons) and bins (large cartons). The objective is to attempt to find the smallest number of bins into which to pack all items. The complexity comes from the fact that there is a large number of candidate bins with different sizes (e.g. 4 to 30) with specific constraints. In addition the time allowed to compute a feasible solution is limited to a few milliseconds. This application is powered by a solver with dedicated heuristics. This is a typical application to deploy on the cloud.

\subsection{Assignment of containers to trains}

This application deals with the operational planning of trains [1] Furthermore, assigning a destination of a train is treated beforehand and the results are inputs for component optimization. The number of wagons and their specifications are also known. The problem is known in literature as "bin packing". The objective is to minimize the number of wagons (bins) while placing the maximum number of containers (items). The application is an interactive Full Web application powered by a CP solver featuring the following functionalities: (1) an optimization engine to compute an initial solution and to assist the operator when completing partial solutions; (2) a fast algorithm to assist the operator in choosing the right container for a wagon.

The constraints taken into account are the maximum weight constraints attached to wagons, the maximum number of containers per wagon, the number of wagons attached to a train and its total weight. Within these limited number of constraints the problem can be solved to optimal degrees. The application handles new additional business constraints, among them: the handling of dangerous goods, the incompatibilities between families of containers, e.g. two containers containing dangerous goods cannot be assigned to the same wagon and the original constraints of wagon-makers which are configuration constraints.

The handling of constraints associated with configurations of the selected wagons make the problem difficult to solve. Therefore bin packing algorithms are enriched and extended to solve variants of cutting stock instances. In addition, one must handle preference constraints to increase the quality of solutions. The use of the cumulative constraint [2] in CP combined with efficient search strategies made it possible to solve the different instances in less than one minute whilst it requires several hours by an operator. One of the interesting features of the application is the possibility to combine $\mathrm{CP}$ and Metaheuristics. The first solver which is $\mathrm{CP}$ based is launched to compute a first correct solution respecting all constraints. The second solver which is based on Metaheuristics assists interactive capabilities of the Full Web application like moving a container from one wagon to another or handling urgent containers.

\section{Handling Curve shapes with CMA-ES algorithms}

This part deals with innovative algorithms in bin packing. The project Net-WMS2, lead by the team Lifeware at INRIA, aims to carry out many studies for complex objects, amongst which solving curve shapes. In addition to packing problems for squares, circles, triangles and polygons [5], we consider continuous packing problems with curve shapes [4]; mixing various shapes and mixing three dimensional packing problems, mixing boxes, spheres and cylinders. On square packing problems, exact methods have been used to find optimal solutions and prove optimality. On curve packing problems, inexact methods like for instance genetic algorithms or hybrid simulated annealing with tabu search methods [7], are usually used to compute suboptimal solutions. In this study, we evaluate the covariance matrix adaptation evolution strategy (CMA-ES2), one of the most powerful evolutionary algorithms for continuous optimization with arbitrary objective functions, on hard packing problems including square shapes, curve shapes, mixed square and curve shapes, and also continuous rotations. The solver made it possible to model and solve a problem in the perfume industry where items are complex shapes defined as Bézier curves. These packing problems are encountered in automotive and perfume industries. The results are promising and details are in [4, 5].

\section{Bin packing and robotics}

Robot packing problems is a new class of bin packing problems requiring dedicated solvers to which we refer to as robot packing algorithms. The problem addressed is solved in two phases. In the first phase the solvers computes an optimal or quasi-optimal solution of the problem to solve. The solution is feasible for operators but in many cases infeasible for robots. The first class of constraint is the placement of items in a box (a carton). The robot's movements are limited. The second class of constraints are known 
as accessibility constraints. Before placing the current item one has to make sure that the placement of remaining items is mechanically feasible. Unfortunately, the handling of these constraints leads to the removal of some conflicting items from the optimal solution.

\section{ARCHITECTURE}

A set of bin packing optimization applications are deployed on the cloud. The design of cloud computing applications requires much care. Some particular applications which are used daily are implemented in such a way to allow the maximum time to optimization algorithms whilst minimizing the exchange time between the server on the cloud and the distant client. Therefore the design of such applications requires deep collaboration between optimization and cloud computing engineers, which are complementary skills. The architecture design is J2EE compliant which is built from the ground up to be a service-oriented architecture (SOA), i.e., it provides most of its business logic encapsulated as middleware services thus enabling third-party applications to be integrated.

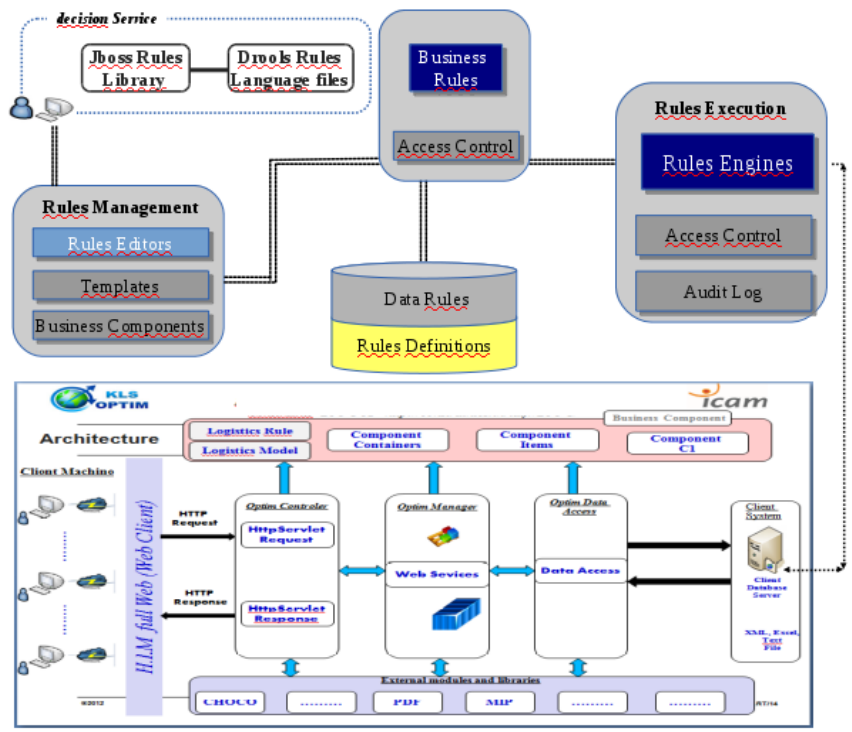

The chosen architecture enables the possibility to integrate the optimisation components in 2-tier architectures (client - server) which are still dominant on the market. Packing applications involve, in fact, several distinct business processes and workflows that require multiple modelling rules and dedicated packing solvers. On the other hand we have the need to use such knowledge and solvers wrapped as a business service provided to distinct consumers. Such consumers may vary from integration with commercial systems (Enterprise Resource Planning, Warehouse Management Systems), specific systems in logistics to end users accessing a web interface application, or external applications invoking the same business logic for their internal use. The fact is that these packing services are required to be exposed and integrated with a set of different systems, users and technologies. Living within a Service Oriented Architecture their interactivity is thus a major concern of the whole decision making application. SOA provides means for composition of services, their interaction and exposure to multiple consumers in a secure and transactional way. The design of a packing application as a composition of services provided by business modules has a consequence on the design and the implementation of optimization algorithms where sharing, common services and data are mandatory principles. Thus, some optimization services exploit capabilities provided by J2EE technologies (AJAX, JQuery, Json, et.); they are tuned making it possible to minimize the data flow (few Kbits) exchanged between the cloud server and the distant client. They take a new turn for the better in efficiency, portability and maintenance. Service-oriented architecture opens new perspectives for agile optimization services to exploit capabilities of RFID and Internet of Things in warehouses and also new applications in robotics, drones, etc.

\section{CONCLUSION}

In this article we have shown the contribution of a variety of algorithms and technologies to solve bin packing problems. Industrial application require robustness and flexibility. $\mathrm{CP}$ is a good candidate tor modeling and solving such problems making a clear distinction between the statement of constraints and the search procedure which can be tuned to compute good quality of solutions. In some cases we have shown where combining $\mathrm{CP}$ and Metaheuristics [1] is more adequate (e.g. assigning containers to wagons). The embedding of optimization solutions in cloud computing environments leads to the organization of optimization algorithms as services opening many perspectives to Metaheuristics and dedicated heuristics to complain with real-time response constraint. Many of the applications described in this article are exploited daily. Items are modeled as regular shapes (1D, 2D and 3D). Over the past few years the demand has been evolving and unfortunately items may have irregular shapes like parts in the automotive industry. Algorithms like grouping items to find bounding boxes (3D) also has its limitation. Solvers based on continuous optimization based on Covariance Matrix Adaptation Evolution Strategy (CMA-ES) opens new perspectives to solve bin packing problems where items are complex shapes which are modeled as Bézier curves and their variants. This approach is more interesting when the solver is integrated as a backend in a declarative modeling language like MiniZinz[6].

\section{ACKNOWLEDGMENTS}

We would like to thank all the partners of the projects Net-WMS-2 (ANR Blanc Simi2) and SOCHART (I-TRANS) for fruitful discussions.

\section{REFERENCES}

[1] Aggoun, A., Rhiat, A., Grassien, J.P., 2011. Online assignments of containers to trains using constraint programming. MIWAI, December 2011.

[2] Aggoun, A., Beldiceanu, N., Bourreau, E. Simonis, H., 1998. The contribution of global constraints for modeling and solving industrial applications. FRANCORO II, Second Journées Francophones Operational Research, Sousse, Tunisia, 1998.

[3] Lodi, A., Martello, S., Monaci, M., 2002. Two Dimensional Packing Problem: A survey. European Journal of Operational Research, Vol. 141, p. 241-252, 2002.

[4] Martinez, T., Fages, F., Aggoun, A., 2015. A Stochastic Continuous Optimization Backend for MiniZinc with Applications to Geometrical Placement Problems. CPAIOR 2016: 13th International Conference on Integration of AI and OR Techniques in Constraint Programming, May 29 - June 1, 2016, Banff, Canada.

[5] Martinez, T., Vitorino, L., Fages, F., Aggoun, A., 2013. On Solving Mixed Shapes Packing Problems by Continuous Optimization with the CMA Evolution Strategy, In Proceedings of the first BRICS countries congress on Computational Intelligence, 2013. 
[6] Nethercote, N., Stuckey, P.J., Becket, R., Brand, S., Duck, G.J., Tack, G.. MiniZinc: Towards a standard CP modelling language. In CP, pages 529-543,2007

[7] Talbi, E-G. Metaheuristics: from design to implementation, Wiley, 2009, ISBN: 978-0-470-27858. 